\title{
Inflammation markers and cognitive performance in breast cancer survivors 20 years after completion of chemotherapy: a cohort study
}

Kimberly D. van der Willik ${ }^{1,2}$, Vincent Koppelmans ${ }^{1,2,3}$, Michael Hauptmann', Annette Compter ${ }^{4}$, M. Arfan kram $^{2}$ and Sanne B. Schagen ${ }^{1,5^{*}}$

\begin{abstract}
Background: Inflammation is an important candidate mechanism underlying cancer and cancer treatment-related cognitive impairment. We investigated levels of blood cell-based inflammatory markers in breast cancer survivors on average 20 years after chemotherapy and explored the relation between these markers and global cognitive performance.

Methods: One hundred sixty-six breast cancer survivors who received post-surgical radiotherapy and six cycles of adjuvant cyclophosphamide, methotrexate, and fluorouracil (CMF) chemotherapy on average 20 years before enrollment were compared with 1344 cancer-free women from a population-based sample (50-80 years old). Breast cancer survivors were excluded if they used adjuvant hormonal therapy or if they developed relapse, metastasis, or second primary malignancies. Systemic inflammation status was assessed by the granulocyte-tolymphocyte ratio (GLR), platelet-to-lymphocyte ratio (PLR), and systemic immune-inflammation index (SII). Cognitive performance was assessed using an extensive neuropsychological test battery from which the general cognitive factor was derived to evaluate global cognitive performance. We examined the association between cancer, the general cognitive factor, and inflammatory markers using linear regression models.

Results: Breast cancer survivors had a lower general cognitive factor than non-exposed participants from the comparator group (mean difference $=-0.21 ; 95 \%$ confidence interval $(\mathrm{Cl})-0.35$ to -0.06 ). Inflammatory markers were higher in cancer survivors compared with non-exposed participants (mean difference for $\log (\mathrm{GLR})=0.31 ; 95 \% \mathrm{Cl} 0.24$ to $0.37, \log (\mathrm{PLR})=0.14 ; 95 \% \mathrm{Cl} 0.09$ to $0.19, \log (\mathrm{SII})=0.31 ; 95 \% \mathrm{Cl} 0.24$ to 0.39$)$. The association between higher levels of inflammatory markers and lower general cognitive factor was statistically significant in cancer survivors but not among non-exposed participants. We found a group-by-inflammatory marker interaction; cancer survivors showed additional lower general cognitive factor per standard deviation increase in inflammatory markers ( $P$ for interaction for $\mathrm{GLR}=0.038, \mathrm{PLR}=0.003$, and $\mathrm{SI}=0.033$ ).

Conclusions: This is the first study to show that (1) cancer survivors have increased levels of inflammation on average 20 years after treatment and (2) these inflammatory levels are associated with lower cognitive performance. Although this association needs verification by a prospective study to determine causality, our findings can stimulate research on the role of inflammation in long-term cognitive problems and possibilities to diminish such problems.
\end{abstract}

Keywords: Breast cancer, Inflammation, Cognitive performance, Cancer/cancer treatment-related side effects

\footnotetext{
* Correspondence: s.schagen@nki.nl

'Department of Psychosocial Research and Epidemiology, Netherlands

Cancer Institute, Plesmanlaan 121, 1066 CX Amsterdam, the Netherlands

${ }^{5}$ Brain and Cognition, Department of Psychology, University of Amsterdam,

Nieuwe Achtergracht 129-B, 1018 WS Amsterdam, the Netherlands

Full list of author information is available at the end of the article
}

(c) The Author(s). 2018 Open Access This article is distributed under the terms of the Creative Commons Attribution 4.0 International License (http://creativecommons.org/licenses/by/4.0/), which permits unrestricted use, distribution, and reproduction in any medium, provided you give appropriate credit to the original author(s) and the source, provide a link to the Creative Commons license, and indicate if changes were made. The Creative Commons Public Domain Dedication waiver (http://creativecommons.org/publicdomain/zero/1.0/) applies to the data made available in this article, unless otherwise stated. 


\section{Background}

Patients with cancer frequently report cognitive problems that can affect their quality of life and daily functioning substantially. Studies have shown that patients with non-central nervous system (non-CNS) cancer can experience cognitive problems during and after completion of treatment including chemotherapy, and a subgroup of patients had cognitive problems up to 20 years after treatment $[1,2]$.

The cancer survivor population is aging and growing because of increased life expectancy and more specifically because of advances in cancer treatment and improved screening. In turn, this results in an increasing number of cancer survivors coping with cognitive problems. The driving forces underlying these cognitive problems have not been sufficiently clarified, impeding the approach and process of developing effective interventions. Cognitive problems in patients with cancer could be induced by cancer itself, cancer-related treatment, or shared risk factors for the development of both cancer and cognitive problems [3, 4]. Disentangling the effects and mechanisms of these causes of disruption of normal cognitive performance is challenging. Different mechanisms, including genetic susceptibility, telomere shortening, changes in hormone levels, and inflammation, have been proposed and revealed [3].

In recent years, inflammation in particular has been suggested as an important and potentially intervenable mechanism in the pathogenesis of cognitive problems in patients with cancer. Higher levels of inflammatory factors such as cytokines are observed in patients with cancer prior to start of any treatment [5], during chemotherapy [6-10], and after chemotherapy [11, 12] up to 5 years after treatment initiation [13]. Several studies found an association between cytokines and cognitive impairment in patients with cancer across different cognitive domains, such as psychomotor speed [8], executive functioning [14], and memory [5, 10, 11, 13]. However, these studies did not agree on the involved cytokines or on the affected cognitive domain. Moreover, because the longest follow-up in these studies was 5 years, it remains unknown whether inflammation also has a role in longer-term or late cognitive problems. Filling this knowledge gap is important as insight into underlying causes of (long-term) cognitive impairment helps to identify those cancer patients at increased risk of developing cognitive problems and opens venues for preventive and therapeutic interventions.

Most studies examined the inflammation status by investigating cytokines using different cytokine panels [5, 6, 8-19]. In contemporary studies, systemic inflammatory response markers measured in blood, including the neutrophil-to-lymphocyte ratio (NLR), platelet-to-lymphocyte ratio (PLR), and systemic immune-inflammation index (SII), are increasingly used. These markers have reliable prognostic and predictive value in patients with cancer and can easily be calculated from readily available standard full blood examination, making them more convenient to use in a clinical setting [20-24]. If related to cognitive problems, these markers could potentially be used as biomarkers for cancer-related cognitive impairment.

In this study, we investigated global cognitive performance, levels of blood cell-based inflammatory markers, and their relation in breast cancer survivors who had received post-surgical radiotherapy and six cycles of adjuvant cyclophosphamide, methotrexate, and fluorouracil (CMF) chemotherapy on average more than 20 years previously. We furthermore examined whether inflammation and cognitive performance were differentially associated between breast cancer survivors and cancer-free women from a population-based sample.

\section{Methods \\ Study population}

In this study, we selected women who had survived breast cancer and had received adjuvant CMF chemotherapy. We compared them with women from the general population, who were cancer-free and had never received chemotherapy.

\section{Breast cancer survivors}

Women with a history of unilateral, invasive breast cancer were identified on the basis of registries of the Netherlands Cancer Institute in Amsterdam and the Daniel den Hoed Cancer Clinic of the Erasmus Medical Center in Rotterdam as described previously [2]. Briefly, women were selected if they had received post-surgical radiotherapy and six cycles of adjuvant CMF chemotherapy between 1976 and 1995.

Breast cancer survivors were eligible if they were 5080 years old at the time of inclusion in 2008, if invasive breast cancer was their first and only malignancy, if they had not developed relapse or distant metastasis, if they had sufficient command of the Dutch language, and if they did not have any contraindications for magnetic resonance imaging (MRI). In addition, ever use of hormonal therapy was applied as an exclusion criterion. Because adjuvant hormonal therapy was not part of the standard treatment for patients with breast cancer in the Netherlands until the mid-1990s, only a few women received this treatment. To enhance homogeneity within the group of breast cancer survivors, we included hormone treatment-naïve cancer survivors only.

Three hundred fifty-nine breast cancer survivors were assessed for eligibility and 292 were selected. Of these 292 women, 196 agreed to participate and provided informed consent. We previously reported on cognitive 
performance of these survivors in comparison with cancer-free women identified within the Rotterdam Study [2]. For the present study, the following additional inclusion criteria were defined: availability of blood measurements and completeness of neuropsychological test data to calculate the general cognitive factor. Thirty of the 196 (15.3\%) breast cancer survivors were excluded because of missing data on blood measurements $(n=5)$ and incomplete data of neuropsychological tests $(n=25$, Fig. 1a). Because breast cancer survivors did not receive an extensive dementia screening, history of dementia was not applied as an exclusion criterion. However, based on the interviews with a trained psychologist, subjective memory complaints, cognitive tests, and brain MRI, it is unlikely that the included breast cancer survivors had dementia at the time of examinations.

\section{Population-based non-exposed participants}

Cancer-free women were selected from the Rotterdam Study, an ongoing population-based prospective cohort that started in 1990 in Rotterdam, the Netherlands. The main objective of the Rotterdam Study is to investigate risk factors of diseases in the elderly. By the end of 2008, the Rotterdam Study consisted of three subcohorts, comprising 14,926 individuals. The design of the Rotterdam Study was described in detail previously [25].

The third subcohort (RS-III) started in 2006 and was the first cohort in which an extensive set of neuropsychological tests was implemented at baseline. For this reason, RS-III was chosen as the reference subcohort, which was composed of 3392 participants (65\% out of invitees). From these participants, women $50-80$ years old without a history of cancer or dementia were eligible as non-exposed participants $(n=1574)$. This sample comprised the non-exposed participants used in our previous cognitive study [2]. Two hundred thirty persons were additionally excluded because of lack of blood measurements $(n=39)$ and incomplete data of neuropsychological tests $(n=191)$, resulting in 1344 non-exposed participants (Fig. 1b).

\section{Assessment of inflammatory markers}

All participants had fasting blood samples taken during the research center visit. Full blood count measurements were performed by using a COULTER Ac.T diff $2^{\text {max }}$ Hematology Analyzer (Beckman Coulter, San Diego, CA, USA) directly after the blood sample was drawn. Hematologic measurements included absolute granulocyte, lymphocyte, and platelet counts in $10^{9}$ per liter.

We used the granulocyte count as proxy for the neutrophil count because we did not have this measurement available in our sample. Because most of the granulocytes are represented by neutrophils, we believe this did not affect our results [26, 27]. For accuracy purposes, we will refer to the granulocyte-to-lymphocyte ratio (GLR) instead of using the term NLR.

The GLR and PLR were calculated as the ratio of granulocyte count to lymphocyte count and as the ratio of platelet count to lymphocyte count, respectively [28]. The SII was defined as platelet count times the GLR [22]. Because they are either ratios or indices, the derived inflammatory markers did not have a unit.

\section{Assessment of cognitive performance}

Cognitive performance was evaluated between November 2009 and June 2010 for breast cancer survivors and between February 2006 and December 2008 for non-exposed participants on the same day the blood sample was drawn. Cognitive performance was assessed by a neuropsychological battery in the research center of the Rotterdam Study. Six tests were administered: the Mini-Mental State Examination, Letter-Digit Substitution Test (LDST), Word Fluency Test (WFT), Stroop Test (reading, naming, and interference), Purdue Pegboard Test (PPB) (right, left, and both hands), and 15-Word Learning Test (15-WLT) (immediate recall, delayed recall, and recognition). Global cognitive
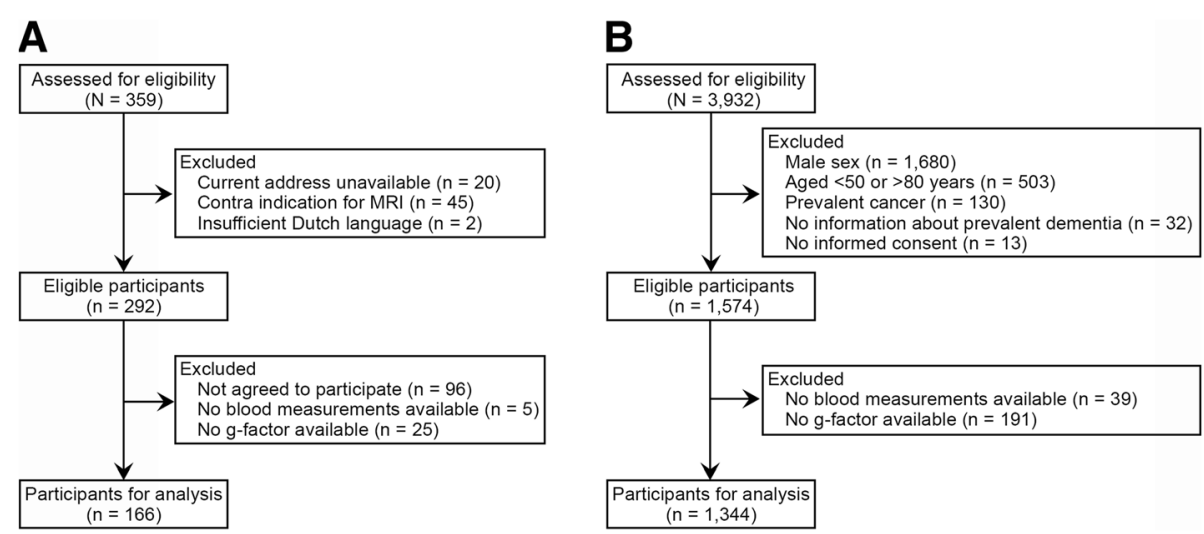

Fig. 1 a Flowchart for breast cancer survivors. b Flowchart for non-exposed participants. Abbreviation: MRI magnetic resonance imaging 
performance was assessed via the general cognitive factor, which was generated by using principal component analysis of the following tests: LDST (total completion time), WFT (number of words), Stroop interference (time in seconds, adjusted for errors), PPB test (total number of pins across three subtasks), and 15-WLT (number of words during delayed recall) [29].

\section{Other assessments}

We assessed education level (primary: primary education; lower: lower general education, intermediate general education, or lower vocational education; intermediate: intermediate vocational education or higher general education; higher: higher vocational education or university) and smoking status (current, former, or never) by interview. Body mass index (BMI) (in kilograms per square meter) was computed from measurements of height and weight. Diabetes mellitus was defined as use of antidiabetic medication, a fasting serum glucose level of at least $7.1 \mathrm{mmol} /$ $\mathrm{L}$, or a random serum glucose level of at least $11.1 \mathrm{mmol} /$ L [30]. History of stroke or myocardial infarction was assessed by interview [31, 32]. Symptoms of depression were evaluated with the Center for Epidemiologic Studies Depression scale (CES-D), which was converted to a sum-score [33]. We had no information about anxiety and fatigue and therefore could not control for these symptoms.

\section{Statistical analyses}

Linear regression models were used to investigate mean differences in the general cognitive factor and inflammatory markers between breast cancer survivors and non-exposed participants. Inflammatory markers were log-transformed because of their skewed distribution. We constructed two nested models: model I was adjusted for age (continuous) and education (four categories), and model II was additionally adjusted for smoking status (three categories), BMI (continuous), diabetes mellitus (yes/no), history of stroke (yes/no), history of myocardial infarction (yes/no), and CES-D sum-score (continuous). To investigate whether levels of the general cognitive factor were explained by different inflammatory markers, we adjusted additionally for each inflammatory marker separately.

The association between the general cognitive factor and inflammatory markers was investigated for breast cancer survivors and non-exposed participants using linear regression models. To study whether this association was stronger in breast cancer survivors than in non-exposed participants, we computed interaction terms between history of cancer/cancer treatment and each inflammatory marker. We explored effect modification by stratifying for mean BMI.
Since mean age was higher in the breast cancer survivors compared with the non-exposed participants (Table 1), we repeated all analyses using age-matched non-exposed participants to minimize residual confounding. These analyses provided estimates comparable to the analyses using all non-exposed participants and therefore are not reported separately.

Multiple imputation was used for missing data on covariates (generally between $0.07 \%$ and $0.3 \%$ with a maximum of $1.8 \%$ for the CES-D sum-score) with five imputed datasets, based on history of cancer/cancer treatment, inflammatory markers, general cognitive factor, and other covariates (that is, age, sex, education, BMI, smoking status, presence of diabetes mellitus, history of stroke, history of myocardial infarction, and CES-D sum-score). Rubin's method was used for pooled regression coefficients $(\beta)$ and $95 \%$ confidence intervals (CIs) [34]. All analyses were performed by using IBM SPSS Statistics Version 24.0 and RStudio Version 3.3.2. All statistical tests were two-sided, and a $P$ value of less than 0.05 was considered statistically significant.

\section{Results}

Characteristics of breast cancer survivors and non-exposed participants are presented in Table 1. Breast cancer survivors were older than non-exposed participants. Additionally, they generally had completed higher levels of education and more often had diabetes mellitus and a history of myocardial infarction. Lastly, although the numbers of never smokers were similar between the two groups, breast cancer survivors were more frequently former smokers and less often current smokers.

\section{Inflammatory markers}

Breast cancer survivors had higher median levels of GLR, PLR, and SII than non-exposed participants. History of breast cancer/cancer treatment was associated with higher inflammatory markers, also after adjustment for age, education, smoking, BMI, diabetes mellitus, history of stroke, history of myocardial infarction, and CES-D sum-score (mean difference for $\log ($ GLR $)=0.31$, $95 \%$ CI 0.24 to $0.37, \log ($ PLR $)=0.14,95 \%$ CI 0.09 to $0.19, \log (\mathrm{SII})=0.31,95 \%$ CI 0.24 to 0.39 ; Table 2$)$. Inflammatory markers were positively associated with age in both groups [35].

\section{Cognitive performance}

Breast cancer survivors had a lower general cognitive factor than non-exposed participants (mean difference $=$ $-0.21,95 \%$ CI -0.35 to -0.06 , corresponding with an effect of 3.6 years of age given a decline in general cognitive factor of 0.59 points per 10 years; Table 2) [29]. Further adjustment for inflammatory factors changed the estimates slightly, indicating that inflammatory 
Table 1 Demographics and characteristics of breast cancer survivors and non-exposed participants

\begin{tabular}{|c|c|c|c|}
\hline Characteristic & $\begin{array}{l}\text { Breast cancer survivors } \\
(n=166)\end{array}$ & $\begin{array}{l}\text { Non-exposed participants } \\
(n=1344)\end{array}$ & $P$ \\
\hline Age in years, mean (SD) & $64.0(6.7)$ & $57.9(5.2)$ & $<0.001$ \\
\hline Education level, no. (\%) & & & $<0.001$ \\
\hline Primary & $14(8.4)$ & $158(11.8)$ & \\
\hline Low & $59(35.5)$ & $616(45.8)$ & \\
\hline Intermediate & $33(19.9)$ & $287(21.4)$ & \\
\hline High & $60(36.1)$ & $283(21.1)$ & \\
\hline Body mass index in $\mathrm{kg} / \mathrm{m}^{2}$, mean (SD) & $26.9(4.6)$ & $27.4(4.8)$ & 0.181 \\
\hline Smoking status, no. (\%) & & & $<0.001$ \\
\hline Current & $16(9.6)$ & $295(21.9)$ & \\
\hline Former & $93(56.0)$ & $574(42.7)$ & \\
\hline Diabetes mellitus, no. (\%) & $14(8.4)$ & $54(4.0)$ & 0.008 \\
\hline History of stroke, no. (\%) & $1(0.6)$ & $19(1.4)$ & 0.715 \\
\hline History of myocardial infarction, no. (\%) & $6(3.6)$ & $11(0.8)$ & 0.001 \\
\hline CES-D sum-score, mean (SD) & $14.5(3.6)$ & $14.8(4.4)$ & 0.450 \\
\hline General cognitive factor, mean (SD) & $-0.39(1.14)$ & $0.05(0.97)$ & $<0.001$ \\
\hline \multicolumn{4}{|l|}{ Inflammatory markers, median (IQR) } \\
\hline GLR & $2.06(1.67-2.66)$ & $1.52(1.20-1.92)$ & $<0.001$ \\
\hline PLR & $145(119-176)$ & $124(102-151)$ & $<0.001$ \\
\hline SII & $618(469-796)$ & $443(328-595)$ & $<0.001$ \\
\hline Age at breast cancer diagnosis in years, mean (SD) & $42.9(5.6)$ & & \\
\hline Time since breast cancer diagnosis, mean (SD) & $21.0(4.5)$ & & \\
\hline
\end{tabular}

Abbreviations: CES-D Center for Epidemiologic Studies Depression Scale, GLR granulocyte-to-lymphocyte ratio, IQR interquartile range, $P L R$ platelet-to-lymphocyte ratio, $S D$ standard deviation, SIl systemic immune-inflammation index

markers explained only a small part of the difference in general cognitive factor in addition to the effect of history of cancer/cancer treatment (mean difference for history of cancer/cancer treatment after adjustment for $\log (\mathrm{GLR})=-0.18 ; 95 \% \mathrm{CI}-0.33$ to $0.02, \log (\mathrm{PLR})=$ $-0.21 ; 95 \% \mathrm{CI}-0.36$ to $0.06, \log (\mathrm{SII})=-0.19 ; 95 \% \mathrm{CI}$ -0.34 to 0.03$)$.

\section{Association between cognitive performance and inflammatory markers by cancer status}

A lower general cognitive factor was associated with higher inflammatory markers in breast cancer survivors (Table 3). In non-exposed participants, higher inflammatory markers tended to be associated with a lower general cognitive factor, albeit not statistically significant.

Table 2 Association between the general cognitive factor and history of cancer and inflammatory markers and history of cancer

\begin{tabular}{lll}
\hline Outcome & Model I & Model II \\
& Mean difference $(95 \% \mathrm{Cl})$ & Mean difference $(95 \% \mathrm{Cl})$ \\
\hline $\begin{array}{l}\text { Inflammatory marker* } \\
\text { Log GLR }\end{array}$ & $0.30(0.24$ to 0.36$)$ & $0.31(0.24$ to 0.37$)$ \\
Log PLR & $0.16(0.10$ to 0.21$)$ & $0.14(0.09$ to 0.19$)$ \\
Log SII & $0.30(0.23$ to 0.38$)$ & $0.31(0.24$ to 0.39$)$ \\
Cognition & \\
General cognitive factor & & $-0.21(-0.35$ to -0.06$)$ \\
\hline
\end{tabular}

Abbreviations: $C l$ confidence interval, GLR granulocyte-to-lymphocyte ratio, PLR platelet-to-lymphocyte ratio, SII systemic immune-inflammation index

Model I is a linear regression of the general cognitive factor or log-transformed inflammatory markers on cancer status adjusted for age and education. Model II is as model I plus adjustment for smoking status, body mass index, diabetes mellitus, history of stroke, history of myocardial infarction, and Center for Epidemiologic Studies Depression Scale (CES-D) sum-score

*Mean difference in general cognitive factor between breast cancer survivors and non-exposed participants

${ }^{\dagger}$ Mean difference in inflammatory markers between breast cancer survivors and non-exposed participants 
Table 3 Association between the general cognitive factor and inflammatory markers in breast cancer survivors and in non-exposed participants

\begin{tabular}{|c|c|c|c|}
\hline \multirow{2}{*}{$\begin{array}{l}\text { Inflammatory } \\
\text { marker per SD } \\
\text { increase }\end{array}$} & \multirow{2}{*}{$\begin{array}{l}\text { Breast cancer survivors } \\
\text { Mean difference* }(95 \% \text { Cl) }\end{array}$} & \multirow{2}{*}{$\begin{array}{l}\text { Non-exposed participants } \\
\text { Mean difference* }(95 \% \mathrm{Cl})\end{array}$} & \multirow{2}{*}{$\begin{array}{l}P \text { for } \\
\text { interaction }\end{array}$} \\
\hline & & & \\
\hline \multicolumn{4}{|l|}{ Model I } \\
\hline $\log G L R$ & $-0.24(-0.40$ to -0.08$)$ & $-0.04(-0.09$ to 0.00$)$ & 0.061 \\
\hline Log PLR & $-0.13(-0.29$ to 0.03$)$ & 0.05 (0.01 to 0.10$)$ & 0.003 \\
\hline Log SII & $-0.22(-0.38$ to -0.07$)$ & $-0.03(-0.08$ to 0.01$)$ & 0.053 \\
\hline \multicolumn{4}{|l|}{ Model II } \\
\hline Log GLR & $-0.23(-0.39$ to -0.08$)$ & $-0.02(-0.07$ to 0.02$)$ & 0.038 \\
\hline Log PLR & $-0.18(-0.33$ to -0.02$)$ & $0.03(-0.01$ to 0.08$)$ & 0.003 \\
\hline Log SII & $-0.23(-0.38$ to -0.07$)$ & $-0.01(-0.06$ to 0.03$)$ & 0.033 \\
\hline
\end{tabular}

Abbreviations: $C l$ confidence interval, $G L R$ granulocyte-to-lymphocyte ratio, $P L R$ platelet-to-lymphocyte ratio, $S D$ standard deviation, $S / l$ systemic immune-inflammation index

Model I is a linear regression of the general cognitive factor on each log-transformed inflammatory marker adjusted for age and education. Model II is as model I plus adjustment for smoking status, body mass index, diabetes mellitus, history of stroke, history of myocardial infarction, and Center for Epidemiologic Studies Depression Scale (CES-D) sum-score

* Mean difference in general cognitive factor per standard deviation increase in inflammatory marker

${ }^{\dagger} P$ value for interaction term between history of cancer/cancer treatment and inflammatory marker

The interaction term between inflammatory markers and history of cancer/cancer treatment was significant for each inflammatory marker, indicating that the association between higher inflammation levels and lower general cognitive factor was more pronounced in breast cancer survivors than in non-exposed participants $(P$ for interaction between cancer and standardized log-transformed GLR $=0.038$, PLR $=0.003$, and SII $=$ 0.033; Fig. 2).

The association between higher inflammatory markers and lower general cognitive factor differed more between breast cancer survivors and non-exposed participants with a higher BMI than in those with a lower BMI. However, stratified analyses for BMI showed that the effect of one-standard deviation increase in inflammatory marker on general cognitive factor was higher among breast cancer survivors with a BMI below $27.3 \mathrm{~kg} / \mathrm{m}^{2}$ compared with those with a higher BMI (Table 4).

\section{Discussion}

This study is the first report investigating the association between blood cell-based inflammatory markers and cognitive performance in breast cancer survivors with an average time since cessation of chemotherapy of more than 20 years. Breast cancer survivors had lower global cognitive performance and higher inflammatory markers compared with women without a history of cancer. The tendency for lower global cognitive performance with higher inflammatory markers was more pronounced in breast cancer survivors, suggesting a potential role for inflammation in the pathophysiology of cognitive problems in cancer
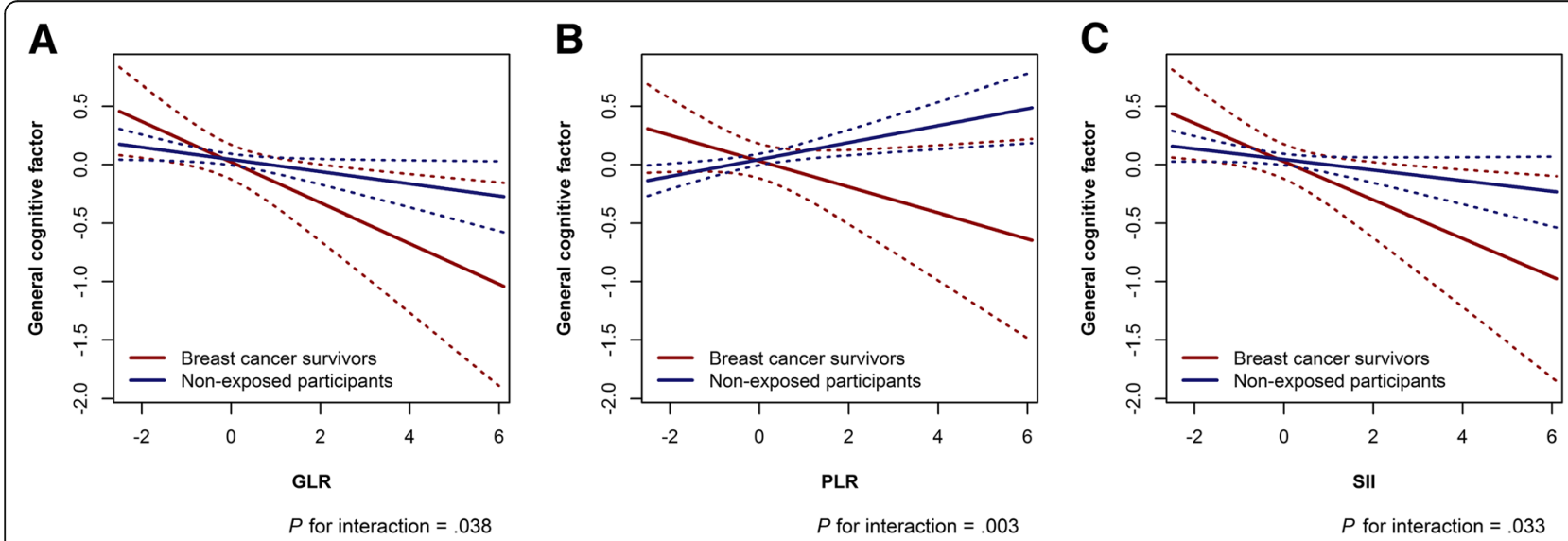

Fig. 2 a Interaction of $\log (G L R)$ and cancer status with the general cognitive factor as outcome. b Same as a, for log (PLR). c Same as a and b, for log(SII). Model used for figure is adjusted for age only. Abbreviations: GLR granulocyte-to-lymphocyte ratio, PLR platelet-to-lymphocyte ratio, SII systemic immune-inflammation index 
Table 4 Association between the general cognitive factor and inflammatory markers in breast cancer survivors and in non-exposed participants stratified for mean body mass index

\begin{tabular}{|c|c|c|c|c|c|}
\hline \multirow{2}{*}{$\begin{array}{l}\text { Inflammatory } \\
\text { marker per } \\
\text { SD increase }\end{array}$} & \multicolumn{2}{|c|}{ Breast cancer survivors } & \multicolumn{2}{|c|}{ Non-exposed participants } & \multirow{2}{*}{$\begin{array}{l}P \text { for } \\
\text { interaction }\end{array}$} \\
\hline & Mean difference ${ }^{*}$ & $95 \% \mathrm{Cl}$ & Mean difference* & $95 \% \mathrm{Cl}$ & \\
\hline \multicolumn{6}{|c|}{$\mathrm{BMl}<27.3 \mathrm{~kg} / \mathrm{m}^{2}$} \\
\hline & $n=104$ & & $n=749$ & & \\
\hline $\log$ GLR & -0.29 & -0.49 to -0.10 & -0.04 & -0.10 to 0.02 & 0.480 \\
\hline Log PLR & -0.22 & -0.42 to -0.02 & 0.01 & -0.05 to 0.08 & 0.309 \\
\hline Log SII & -0.28 & -0.48 to -0.09 & -0.04 & -0.10 to 0.02 & 0.564 \\
\hline \multicolumn{6}{|c|}{$\mathrm{BMI}>27.3 \mathrm{~kg} / \mathrm{m}^{2}$} \\
\hline & $n=62$ & & $n=595$ & & \\
\hline $\log$ GLR & -0.16 & -0.41 to 0.09 & 0.01 & -0.06 to 0.08 & 0.013 \\
\hline Log PLR & -0.16 & -0.42 to 0.10 & 0.05 & -0.02 to 0.12 & $<0.001$ \\
\hline Log SII & -0.12 & -0.38 to 0.14 & 0.02 & -0.05 to 0.09 & 0.005 \\
\hline
\end{tabular}

Abbreviations: $B M I$ body mass index, $C I$ confidence interval, GLR granulocyte-to-lymphocyte ratio, $P L R$ platelet-to-lymphocyte ratio, $S D$ standard deviation, $S I I$ systemic immune-inflammation index

Model I is a linear regression of the general cognitive factor on each log-transformed inflammatory marker adjusted for age and education. Model II is as model I plus adjustment for smoking status, diabetes mellitus, history of stroke, history of myocardial infarction, and Center for Epidemiologic Studies Depression Scale (CES-D) sum-score.

*Mean difference in general cognitive factor per standard deviation increase in inflammatory marker

${ }^{\dagger} P$ value for interaction term between history of cancer/cancer treatment and inflammatory marker

survivors. This effect was not modified by BMI. More insight in mechanisms underlying cognitive problems could help identifying those women who are at an increased risk of cognitive problems and developing prevention strategies.

We previously reported on differences in cognitive performance between breast cancer survivors and non-exposed participants [2]. In this previous study, we tested between-group performance differences of individual cognitive outcome measures that were currently used to construct the general cognitive factor and observed that breast cancer survivors performed worse compared with non-exposed participants within several cognitive domains. This suggested that cognitive problems in cancer survivors can be long-lasting. In the present study, we evaluated global cognitive performance using the general cognitive factor because we did not expect a specific cognitive domain to be affected by inflammation. We chose to use a robust cognitive summary measure, thereby reducing the number of comparisons.

Interestingly, levels of inflammatory markers were higher in breast cancer survivors, compared with non-exposed participants, on average 20 years after cancer treatment. Inflammation plays a critical role in tumorigenesis, tumor progression, and cancer metastasis $[36,37]$. Research has shown that chronic inflammation is associated with an increased cancer risk [37]. Moreover, different markers of inflammation, such as cytokines, C-reactive protein, and NLR, are often elevated in patients with cancer and are associated with poor survival $[9,15-17,24,38]$. One study investigating inflammation levels after cancer treatment found that C-reactive protein and cytokine levels were elevated up to 5 years after treatment [19]. Our observation that systemic inflammation markers are higher in breast cancer survivors compared with non-exposed participants on average 20 years after cancer treatment suggests deregulation of the immune system. Whether this is a consequence of cancer or cancer treatment (or both) or a pre-existing deregulation before cancer development cannot be determined with the present study.

The found association of blood cell-based inflammatory markers and cognitive performance in breast cancer survivors is in line with previous observations before, during, and shortly after therapy $[6,17,18]$. Two studies investigated the link between inflammation and cognitive performance prior to the start of cancer treatment. The first study showed that elevated levels of interleukin-6 (IL-6) in patients with acute myelogenous leukemia or myelodysplastic syndrome were associated with poorer executive functioning before cancer treatment [14]. The second study showed that high levels of soluble tumor necrosis factor receptor type II (sTNF-RII) were related to reduced verbal memory performance in patients with newly diagnosed breast cancer [5]. More studies in patients with breast cancer have tried to elucidate the role of inflammation in impaired cognitive performance during chemotherapy and two of these studies identified specific cytokines to be involved. Williams et al. focused on sTNR-RII and found that higher levels of this receptor were associated with visual memory performance [10]. Cheung et al. observed an association between increased levels of IL- 6 and IL-1 $\beta$ 
and poorer psychomotor speed performance during chemotherapy [8]. Shortly after cancer treatment, higher levels of sTNF-RII were associated with increased memory complaints [11], and on average 5 years after cancer treatment, elevated IL- 6 and TNF $\alpha$ levels were associated with worse verbal memory [13]. Importantly, the association between inflammation and cognitive performance is supported by animal studies. Acute peripheral immune challenges using lipopolysaccharide resulted in cognitive impairments in a spatial working memory task in mice. Cognitive impairments were observed $1.5-2 \mathrm{~h}$ after injection in tumor-bearing mice but not in tumor-free mice. These cognitive effects could be prevented when using a technique to enhance innate immune reactivity [39]. Together, these results support the hypothesis that inflammation has a role in the complex pathogenesis of both short-term and longer-term cognitive problems in patients with cancer.

Owing to our study design, we cannot determine whether the association between inflammation and impaired cognitive performance is causal. However, also a causal association could not illuminate the exact underlying mechanisms by which inflammation leads to brain changes and subsequent cognitive problems. Peripheral pro-inflammatory cytokines are able to cross the bloodbrain barrier, which may initiate the release of local cytokines [40]. Local cytokine production could result in neurotransmitter deregulation, increased oxidative stress, and decreased neurogenesis and neuroplasticity, which in turn can lead to cognitive dysfunction [41]. It is also possible that inflammation induces epigenetic changes and chromosomal instability, which can be persistent and therefore could be associated with long-term cognitive problems [42].

Our study has several strengths. First, we have a large sample size of breast cancer survivors who have been treated on average more than 20 years ago, enabling us to investigate long-term effects. Moreover, we used non-exposed participants from a population-based cohort study, who underwent the same examinations as the breast cancer survivors. This design provided standardized ascertainments of outcome and covariates. All participants received a neuropsychological test battery, enabling us to investigate global cognitive function by the general cognitive factor. Lastly, we were able to investigate inflammation status using blood cell-based inflammatory markers, which are low-cost and easy to use in the clinic.

Study limitations include the design by which we cannot disentangle the effects of cancer and cancer treatment on cognition and levels of inflammatory markers. Some studies show that patients treated with chemotherapy have higher inflammatory markers during and after treatment compared with chemotherapy-naïve patients [12]. However, because inflammatory markers and cognitive problems can already occur in patients with newly diagnosed cancer, it is unlikely that inflammation is important only in chemotherapytreated patients [5]. Owing to the cross-sectional design, we do not have information about cognitive performance and levels of inflammatory markers before cancer diagnosis and treatment. Moreover, patients with breast cancer nowadays receive chemotherapy regimens other than CMF, either with or without adjuvant endocrine therapy, limiting the generalizability to current patients with breast cancer.

However, cyclophosphamide and 5-fluoroacil are still frequently used in other regimens for adjuvant chemotherapy. Furthermore, we were not able to exclude individuals whose systemic inflammatory markers may have been elevated because of acute infections and to control for acute-phase reactants such as C-reactive protein, but we expect that this effect is similar for cancer survivors and non-exposed participants. Lastly, we need to emphasize that by measuring the GLR, PLR, and SII, we cannot identify the exact phenotype of the underlying immune cell populations. Although these markers are proven to be related to chronic systemic inflammation, it is unknown whether they also reflect higher levels of pro-inflammatory cytokines. In other words, we cannot confirm that observed shifts in the granulocytes, lymphocytes, and platelets cause higher cytokine levels and thereby are functional. To elucidate the exact immune cell populations involved in increases of the GLR, PLR, and SII, determination of different cytokines is needed.

\section{Conclusions}

We found that breast cancer survivors who had been treated with chemotherapy on average more than 20 years ago have higher blood cell-based inflammatory markers compared with women without a history of cancer. Higher levels of inflammatory markers tended to be associated with poorer cognitive performance in both cancer survivors and cancer-free women, and expression was stronger in breast cancer survivors. This finding suggests that inflammation could have a role in the pathogenesis of long-term cognitive impairment in cancer survivors. Further prospective studies are important to determine the causality of the association and to investigate the effects of lowering inflammation on the development of cognitive problems in cancer patients and survivors, for instance, by exercise or anti-inflammatory drugs.

\footnotetext{
Abbreviations

15-WLT: 15-Word Learning Test; BMI: Body mass index; CES-D: Center for Epidemiologic Studies Depression Scale; Cl: Confidence interval;

CMF: Cyclophosphamide, methotrexate, and fluorouracil; GLR: Granulocyteto-lymphocyte ratio; IL: Interleukin; LDST: Letter-Digit Substitution Test; MRI: Magnetic resonance imaging; NLR: Neutrophil-to-lymphocyte ratio; PLR: Platelet-to-lymphocyte ratio; PPB: Purdue Pegboard Test; RS-III: Third subcohort of the Rotterdam Study; SIl: Systemic immune-inflammation index; sTNF-RII : Soluble tumor necrosis factor receptor type II; TNF: Tumor necrosis factor; WFT: Word Fluency Test
} 


\section{Acknowledgments}

We gratefully thank all participants and staff for their time and commitment to the study. The authors are grateful for the intellectual contributions that K.E. de Visser, Division of Immunology, Netherlands Cancer Institute, made to this study.

\section{Funding}

This work was supported by the Dutch Cancer Society (grant number NKI20157737). The Rotterdam Study is funded by Erasmus Medical Center and Erasmus University, Rotterdam, Netherlands Organization for the Health Research and Development (ZonMw), the Research Institute for Diseases in the Elderly (RIDE), the Ministry of Education, Culture and Science, the Ministry for Health, Welfare and Sports, the European Commission (DG XII), and the Municipality of Rotterdam. The funders had no role in study design, data collection and analysis, decision to publish, or preparation of the manuscript.

\section{Availability of data and materials}

Data can be obtained upon request. Requests should be directed toward the management team of the Rotterdam Study (secretariat.epi@erasmusmc.nl), which has a protocol for approving data requests. Because of restrictions based on privacy regulations and informed consent of the participants, data cannot be made freely available in a public repository. The Rotterdam Study has been approved by the Medical Ethics Committee of the Erasmus MC (registration number MEC 02.1015) and by the Dutch Ministry of Health, Welfare and Sport (Population Screening Act WBO, license number 1071272 159521-PG). The Rotterdam Study has been entered into the Netherlands National Trial Register (www.trialregister.nl) and into the WHO International Clinical Trials Registry Platform (www.who.int/ictrp/network/primary/en/) under shared catalogue number NTR6831. All participants provided written informed consent to participate in the study and to have their information obtained from treating physicians.

\section{Authors' contributions}

$\mathrm{KDW}, \mathrm{SBS}$, and MAI contributed to study design. MAI and SBS contributed to funding. VK contributed to data collection. KDW contributed to data analysis. All authors contributed to data interpretation and critical revision and approved the final manuscript.

\section{Ethics approval and consent to participate}

Breast cancer survivors: Written informed consent was obtained from all participants. The institutional review boards of the two participating institutions (the Netherlands Cancer Institute/Antoni van Leeuwenhoek Hospital and the Erasmus University Medical Center) approved the study. Rotterdam Study: The Rotterdam Study has been approved by the Medical Ethics Committee of the Erasmus MC (registration number MEC 02.1015) and by the Dutch Ministry of Health, Welfare and Sport (Population Screening Act WBO, license number 1071272-159521-PG). The Rotterdam Study has been entered into the Netherlands National Trial Register (www.trialregister.nl) and the WHO International Clinical Trials Registry Platform (www.who.int/ictrp/network/primary/en/) under shared catalogue number NTR6831. All participants provided written informed consent to participate in the study and to have their information obtained from treating physicians.

\section{Consent for publication}

Not applicable.

\section{Competing interests}

The authors declare that they have no competing interests.

\section{Publisher's Note}

Springer Nature remains neutral with regard to jurisdictional claims in published maps and institutional affiliations.

\section{Author details}

'Department of Psychosocial Research and Epidemiology, Netherlands Cancer Institute, Plesmanlaan 121, 1066 CX Amsterdam, the Netherlands. 2Department of Epidemiology, Erasmus MC - University Medical Center Rotterdam, PO Box 2040, 3000 CA Rotterdam, the Netherlands. ${ }^{3}$ Department of Psychiatry, The University of Utah, 501 Chipeta Way, Salt Lake City, UT 84108, USA. ${ }^{4}$ Department of Neuro-oncology, Netherlands Cancer Institute,
Plesmanlaan 121, 1066 CX Amsterdam, the Netherlands. ${ }^{5}$ Brain and Cognition, Department of Psychology, University of Amsterdam, Nieuwe Achtergracht 129-B, 1018 WS Amsterdam, the Netherlands.

Received: 7 June 2018 Accepted: 12 October 2018

Published online: 15 November 2018

\section{References}

1. Janelsins MC, Kesler SR, Ahles TA, Morrow GR. Prevalence, mechanisms, and management of cancer-related cognitive impairment. Int Rev Psychiatry. 2014:26:102-13.

2. Koppelmans V, Breteler MM, Boogerd W, Seynaeve C, Gundy C, Schagen SB. Neuropsychological performance in survivors of breast cancer more than 20 years after adjuvant chemotherapy. J Clin Oncol. 2012;30:1080-6.

3. Ahles TA, Saykin AJ. Candidate mechanisms for chemotherapy-induced cognitive changes. Nat Rev Cancer. 2007;7:192-201.

4. Ahles TA, Root JC, Ryan EL. Cancer- and cancer treatment-associated cognitive change: an update on the state of the science. J Clin Oncol. 2012;30:3675-86.

5. Patel SK, Wong AL, Wong FL, Breen EC, Hurria A, Smith $M$, et al. Inflammatory Biomarkers, Comorbidity, and Neurocognition in Women With Newly Diagnosed Breast Cancer. J Natl Cancer Inst. 2015;107.

6. Lyon DE, Cohen R, Chen H, Kelly DL, McCain NL, Starkweather A, et al. Relationship of systemic cytokine concentrations to cognitive function over two years in women with early stage breast cancer. J Neuroimmunol. 2016;301:74-82.

7. Briones TL, Woods J. Dysregulation in myelination mediated by persistent neuroinflammation: possible mechanisms in chemotherapy-related cognitive impairment. Brain Behav Immun. 2014;35:23-32.

8. Cheung YT, Ng T, Shwe M, Ho HK, Foo KM, Cham MT, et al. Association of proinflammatory cytokines and chemotherapy-associated cognitive impairment in breast cancer patients: a multi-centered, prospective, cohort study. Ann Oncol. 2015;26:1446-51.

9. Pusztai L, Mendoza TR, Reuben JM, Martinez MM, Willey JS, Lara J, et al. Changes in plasma levels of inflammatory cytokines in response to paclitaxel chemotherapy. Cytokine 2004;25:94-102.

10. Williams AM, Shah R, Shayne M, Huston AJ, Krebs M, Murray N, et al. Associations between inflammatory markers and cognitive function in breast cancer patients receiving chemotherapy. J Neuroimmunol. 2018;314:17-23.

11. Ganz PA, Bower JE, Kwan L, Castellon SA, Silverman DH, Geist C, et al. Does tumor necrosis factor-alpha (TNF-alpha) play a role in post-chemotherapy cerebral dysfunction? Brain Behav Immun. 2013;30(Suppl):S99-108.

12. Pomykala KL, Ganz PA, Bower JE, Kwan L, Castellon SA, Mallam S, et al. The association between pro-inflammatory cytokines, regional cerebral metabolism, and cognitive complaints following adjuvant chemotherapy for breast cancer. Brain Imaging Behav. 2013;7:511-523.

13. Kesler S, Janelsins M, Koovakkattu D, Palesh O, Mustian K, Morrow G, et al. Reduced hippocampal volume and verbal memory performance associated with interleukin-6 and tumor necrosis factor-alpha levels in chemotherapytreated breast cancer survivors. Brain Behav Immun. 2013;30(Suppl):S109-16.

14. Meyers CA, Albitar M, Estey E. Cognitive impairment, fatigue, and cytokine levels in patients with acute myelogenous leukemia or myelodysplastic syndrome. Cancer. 2005;104:788-93.

15. Janelsins MC, Mustian KM, Palesh OG, Mohile SG, Peppone LJ, Sprod LK, et al. Differential expression of cytokines in breast cancer patients receiving different chemotherapies: implications for cognitive impairment research. Support Care Cancer. 2012;20:831-9.

16. Lippitz BE. Cytokine patterns in patients with cancer: a systematic review. Lancet Oncol. 2013;14:e218-28.

17. Seruga B, Zhang H, Bernstein $\sqcup$, Tannock IF. Cytokines and their relationship to the symptoms and outcome of cancer. Nat Rev Cancer. 2008;8:887-99.

18. Wang XS, Shi Q, Williams LA, Mao L, Cleeland CS, Komaki RR, et al. Inflammatory cytokines are associated with the development of symptom burden in patients with NSCLC undergoing concurrent chemoradiation therapy. Brain Behav Immun. 2010;24:968-74.

19. Vardy JL, Booth C, Pond GR, Zhang H, Galica J, Dhillon H, et al. Cytokine levels in patients (pts) with colorectal cancer and breast cancer and their relationship to fatigue and cognitive function. Suppl J Clin Oncol. 2007;18S:9070.

20. Templeton AJ, McNamara MG, Seruga B, Vera-Badillo FE, Aneja P, Ocana A, et al. Prognostic role of neutrophil-to-lymphocyte ratio in solid tumors: a systematic review and meta-analysis. J Natl Cancer Inst. 2014;106:dju124. 
21. Kwon HC, Kim SH, Oh SY, Lee S, Lee JH, Choi HJ, et al. Clinical significance of preoperative neutrophil-lymphocyte versus platelet-lymphocyte ratio in patients with operable colorectal cancer. Biomarkers. 2012;17:216-22.

22. Hu B, Yang XR, Xu Y, Sun YF, Sun C, Guo W, et al. Systemic immuneinflammation index predicts prognosis of patients after curative resection for hepatocellular carcinoma. Clin Cancer Res. 2014;20:6212-22.

23. Ethier JL, Desautels D, Templeton A, Shah PS, Amir E. Prognostic role of neutrophil-to-lymphocyte ratio in breast cancer: a systematic review and meta-analysis. Breast Cancer Res. 2017;19:2.

24. Chen J, Deng Q, Pan Y, He B, Ying H, Sun H, et al. Prognostic value of neutrophilto-lymphocyte ratio in breast cancer. FEBS Open Bio. 2015;5:502-507.

25. Ikram MA, Brusselle GGO, Murad SD, van Duijn $\mathrm{CM}$, Franco $\mathrm{OH}$, Goedegebure A, et al. The Rotterdam Study: 2018 update on objectives, design and main results. Eur J Epidemiol. 2017;32:807-50.

26. Patton KT, Thibodeau GA. Anatomy and Physiology. 9th ed. St Louis: Elsevier; 2016.

27. Wulaningsih W, Holmberg L, Abeler-Doner L, Ng T, Rohrmann S, Van Hemelrijck M. Associations of C-Reactive Protein, Granulocytes and Granulocyte-to-Lymphocyte Ratio with Mortality from Breast Cancer in NonInstitutionalized American Women. PLoS One. 2016;11:e0157482.

28. He W, Yin C, Guo G, Jiang C, Wang F, Qiu H, et al. Initial neutrophil lymphocyte ratio is superior to platelet lymphocyte ratio as an adverse prognostic and predictive factor in metastatic colorectal cancer. Med Oncol. 2013;30:439.

29. Hoogendam YY, Hofman A, van der Geest JN, van der Lugt A, Ikram MA Patterns of cognitive function in aging: the Rotterdam Study. Eur J Epidemiol. 2014:29:133-40.

30. Diabetes mellitus. Report of a WHO Study Group. World Health Organ Tech Rep Ser. 1985;727:1-113.

31. Bos MJ, Koudstaal PJ, Hofman A, Ikram MA. Modifiable etiological factors and the burden of stroke from the Rotterdam study: a population-based cohort study. PLoS Med. 2014;11:e1001634.

32. Hak AE, Pols HA, Visser TJ, Drexhage HA, Hofman A, Witteman JC. Subclinical hypothyroidism is an independent risk factor for atherosclerosis and myocardial infarction in elderly women: the Rotterdam Study. Ann Intern Med. 2000;132:270-8.

33. Mirza SS, de Bruijn RF, Direk N, Hofman A, Koudstaal PJ, Ikram MA, et al. Depressive symptoms predict incident dementia during short- but not longterm follow-up period. Alzheimers Dement. 2014;10(5 Suppl):S323-9 e321.

34. Rubin DB. Multiple imputation for nonresponse in surveys. New York: Wiley; 1987.

35. Lin BD, Hottenga JJ, Abdellaoui A, Dolan CV, de Geus EJ, Kluft C, et al. Causes of variation in the neutrophil-lymphocyte and platelet-lymphocyte ratios: a twin-family study. Biomark Med. 2016 [Epub ahead of print].

36. Coussens LM, Werb Z. Inflammation and cancer. Nature. 2002;420:860-7.

37. Shacter E, Weitzman SA. Chronic inflammation and cancer. Oncology (Williston Park). 2002;16:217-26 229; discussion 230-212.

38. Pierce BL, Ballard-Barbash R, Bernstein L, Baumgartner RN, Neuhouser ML, Wener $\mathrm{MH}$, et al. Elevated biomarkers of inflammation are associated with reduced survival among breast cancer patients. J Clin Oncol. 2009;27:3437-44.

39. Bever SR, Liu X, Quan N, Pyter LM. Euflammation Attenuates Central and Peripheral Inflammation and Cognitive Consequences of an Immune Challenge after Tumor Development. Neuroimmunomodulation. 2017;24:74-86.

40. Ren X, St Clair DK, Butterfield DA. Dysregulation of cytokine mediated chemotherapy induced cognitive impairment. Pharmacol Res. 2017;117:267-73.

41. Wang XM, Walitt B, Saligan L, Tiwari AF, Cheung CW, Zhang ZJ. Chemobrain: a critical review and causal hypothesis of link between cytokines and epigenetic reprogramming associated with chemotherapy. Cytokine. 2015;72:86-96.

42. Lyon D, Elmore L, Aboalela N, Merrill-Schools J, McCain N, Starkweather A, et al. Potential epigenetic mechanism(s) associated with the persistence of psychoneurological symptoms in women receiving chemotherapy for breast cancer: a hypothesis. Biol Res Nurs. 2014;16:160-74.

Ready to submit your research? Choose BMC and benefit from:

- fast, convenient online submission

- thorough peer review by experienced researchers in your field

- rapid publication on acceptance

- support for research data, including large and complex data types

- gold Open Access which fosters wider collaboration and increased citations

- maximum visibility for your research: over $100 \mathrm{M}$ website views per year

At BMC, research is always in progress.

Learn more biomedcentral.com/submissions 